\title{
Investigation into nutrition and health claims of weight management products, specifically meal replacement for weight control bars, compliance with EU regulations and a survey of consumer understanding
}

\author{
E. Vivante ${ }^{1}$, W.L. Hall ${ }^{1}$ and K.L. Johnston ${ }^{2}$ \\ ${ }^{1}$ Department of Nutritional Sciences, School of Life Course Sciences, King's College London, London, UK and \\ ${ }^{2}$ Lighterlife (UK) Ltd, Cavendish House, Harlow, UK
}

The EU strictly regulates communication of nutrition and health claims (NHCs). Regulation (EC) No 1924/2006 ${ }^{(1)}$ provides the legal framework on NHCs. However, which NHCs can be used by manufacturers and what they mean to consumers can be a source of confusion. This study focuses on meal replacement for weight control bars (MRBs), ultimately a type of weight management product, sold as general food with on-package information such as NHCs. This study aims to investigate whether NHCs used by companies that sell meal replacements for weight control in the form of bars, e.g. cereal or paste bars (MRBs) sold to consumers in the UK comply with EU law. A further aim is to explore consumer understanding of the meaning of NHCs.

Online horizon scanning was performed to identify all MRB brands available to consumers in the UK. Product labelling was assessed regarding compliance with EU regulations. Finally, a questionnaire was designed and administered online to volunteers aged $18+$ by convenience sampling to determine their understanding of selected on-package claims. Ethical approval was obtained from King's College London Research Ethics Committee.

Of 21 MRB brands identified, 10 did not comply with EU regulations on NHCs. Non-compliance was linked to the use of unauthorised NHCs, failure to meet labelling requirements, compositional criteria or the provision of incomplete information. The consumer understanding survey had 107 respondents. 75\% of respondents reported understanding the nutrition claim (NC) "high fibre" and 80\% "high protein". Health claims (HCs) were less understood: $49 \%$ reported understanding the HC "Substituting two daily meals of an energy-restricted diet with meal replacements contributes to weight loss" and 44\% reported understanding "Substituting one or two daily meals with meal replacements helps to maintain body weight after weight loss". In addition, the questionnaire included images of two MRBs: $57 \%$ of participants were able to identify a NC and only $28 \%$ of participants recognized that no $\mathrm{HC}$ was present.

All MRB brands used NHCs, almost half were not EU regulation compliant. Manufacturer compliance improvement requires both access to enhanced information, especially post Brexit, and increased enforcement. Self-reported consumer understanding of HCs for weight loss and weight maintenance was low in comparison to NCs, possibly due to lack of familiarity and credibility. Considering the expected strong consumption growth of these products, further research is warranted.

\section{Acknowledgments}

I would like to express my gratitude to my supervisors Dr Wendy Hall and Dr Kelly Johnston for their expertise, help, patience and support throughout this research project

\section{Reference}

1. EC (2006) the OJEU, L404/9. 